\title{
Simple covering technique in thoracoscopic bullectomy for spontaneous pneumothorax
}

\author{
Manabu Yasuda ${ }^{1}$, Ryoichi Nakanishi ${ }^{2}$, Masataka Mori ${ }^{1}$, Syuhei Ashikari ${ }^{1}$, Tsunehiro Oyama $^{1}$, Takeshi \\ Hanagiri $^{1}$ \\ ${ }^{1}$ Department of Thoracic Surgery, Shin-Kokura Hospital, Federation of National Public Service, Personnel Mutual Aid Associations, Kitakyushu, \\ Japan; ${ }^{2}$ Department of Oncology, Immunology and Surgery, Graduate School of Medical Sciences and Medical School, Nagoya City University, \\ Nagoya, Japan \\ Correspondence to: Manabu Yasuda, MD, PhD. Department of Thoracic Surgery, Shin-Kokura Hospital, Federation of National Public Service, \\ Personnel Mutual Aid Associations,1-3-1 Kanada, Kokurakita-ku, Kitakyushu 803-8505, Japan. Email: myasudah3@aih-net.com.
}

\begin{abstract}
Reduced-port thoracoscopic surgery for pneumothorax has been well reported. However, the optimum method for preventing postoperative recurrence in reduced-port thoracoscopic bullectomy remains unclear. We investigated ways to improve the covering technique with reduced-port thoracoscopic bullectomy for spontaneous pneumothorax. From April to December 2016, we performed a simple covering technique with reduced-port thoracoscopic bullectomy on six patients and evaluated the surgical results and patient outcomes. All of the patients were successfully treated with the simple covering technique, and none had a postoperative ipsilateral recurrence of pneumothorax. Our method is a simple and easy technique involving the wide reinforcement of the staple line and may be effective in reducing the risk of postoperative recurrence in reduced-port thoracoscopic bullectomy.
\end{abstract}

Keywords: Reduced-port thoracoscopic bullectomy; staple-line reinforcement; wide covering technique

Submitted Apr 09, 2017. Accepted for publication Aug 24, 2017.

doi: $10.21037 /$ jtd.2017.10.95

View this article at: http://dx.doi.org/10.21037/jtd.2017.10.95

\section{Introduction}

Because of its decreased postoperative pain, shorter duration of chest insertion and consequently shorter hospital stays, reduced-port video-assisted thoracoscopic surgery (VATS) is considered a technically feasible option for many types of pulmonary surgery given recent developments in thoracoscopic instruments and techniques (1). For spontaneous pneumothorax, thoracic surgeons usually apply a reduced-port thoracoscopic approach $(2,3)$. However, the optimum method for preventing postoperative recurrence in reduced-port thoracoscopic bullectomy remains unclear.

Several reports have found that patients who receive resection of bullae alone in VATS are at a high risk of postoperative recurrence (4). Many surgeons have therefore examined the utility of an additional coverage procedure on the staple line to prevent recurrent pneumothorax after thoracoscopic bullectomy (5). Saito et al. reported a covering technique involving resection by endostapling across the covering polymeric biodegradable sheet (6). We herein report a modified version of that technique via a reduced-port thoracoscopic approach as well as the surgical results and patient outcomes.

\section{Operative techniques}

From April to December 2016, we performed thoracoscopic bullectomy by reduced-port VATS on six patients, at the Department of Thoracic Surgery, Shin-Kokura Hospital, Federation of National Public Service, Personnel Mutual Aid Associations, Japan. The local Research Ethics Committee approved the study, and all patients gave their written consent.

The technique (the jellyfish method) was as follows (Figures 1 and 2): a 1.5 - to $2.0-\mathrm{cm}$ incision was made in the skin at the fourth intercostal space in the anterior 
$A_{(a)}$

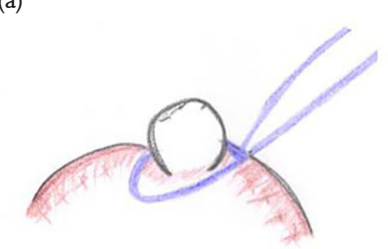

(c)

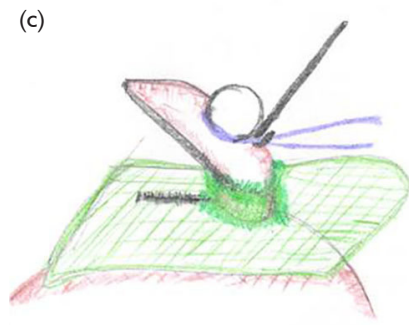

(e)

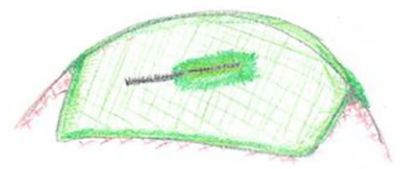

(b)

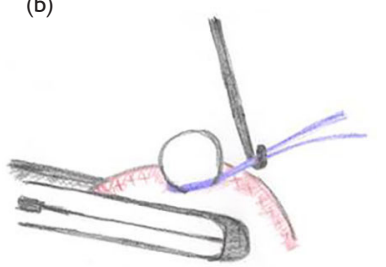

(d)

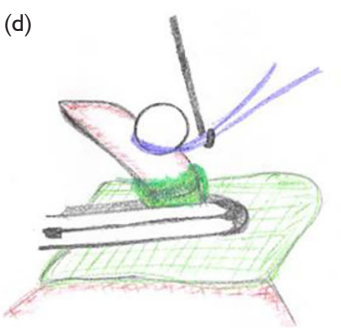

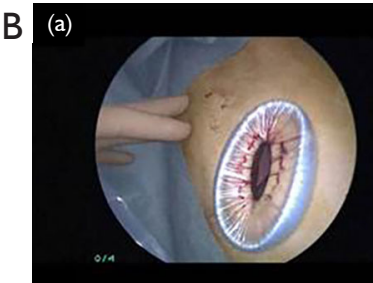

(c)

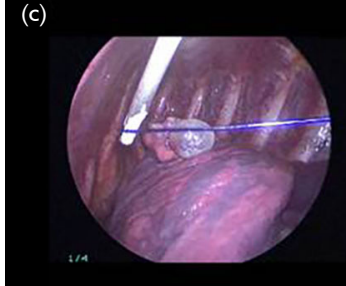

(e)

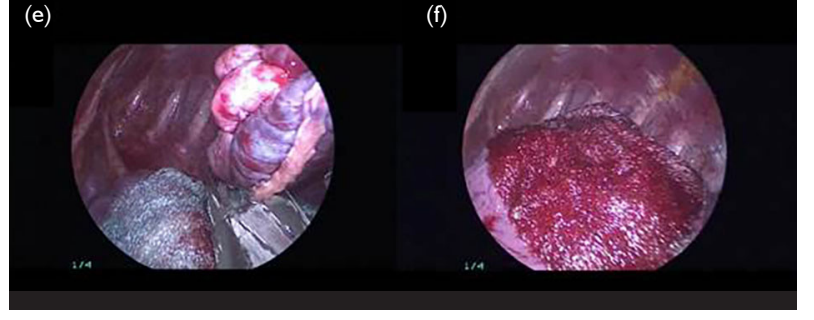

Figure 1 The jellyfish method was as follows (Case No. 1): (A) (a) the bullous lesion was ligated using an endoloop; (b) endostapling was done for the ligated bullous lesion first; (c) the ligated bullous lesion was then pulled through the PGA sheet; (d) the lesion was resected by endostapling across the PGA sheet; (e) the covered PGA sheet was fixed at the staple line. (B) (a) A 1.5- to 2.0-cm incision was made in the skin at the fourth intercostal space in the anterior axillary line, and a wound protector was tightly put in place; (b) the bullous lesion was ligated using an endoloop; (c) a thin puncture device was then inserted through an additional percutaneous route, and the ligated lesion was lifted carefully; (d) the ligated bullous lesion was pulled through the PGA sheet; (e) the lesion was resected by endostapling across the PGA sheet; (f) autologous blood was placed drop-wise onto the sheet as an autologous blood patch. PGA, polyglycolic acid.

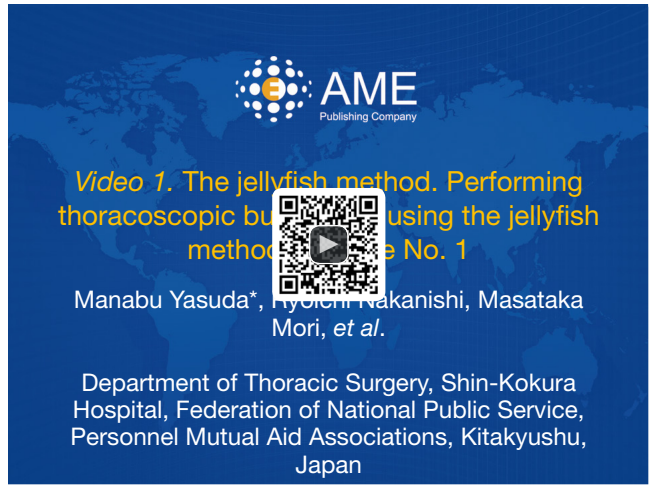

Figure 2 The jellyfish method. Performing thoracoscopic bullectomy using the jellyfish method for Case No. 1 (7).

Available online: http://www.asvide.com/articles/1836

axillary line, and a wound protector was tightly put in place (SurgiSleeve XS Wound Protector, COVIDIEN Japan, Tokyo, Japan). A 5-mm $30^{\circ}$ video thoracoscope combined with 5-mm thoracoscopic forceps or 6.4-mm Thoraco Cotton (KENZMEDICO Co., Saitama, Japan) was introduced into the thoracic cavity through the access window. First, the bullous lesion was ligated by using an endoloop (ENDOLOOP; ETHICON Japan, Tokyo, Japan). A thin puncture device (Endo Close; COVIDIEN Japan, Tokyo, Japan) was then inserted through an additional percutaneous route, and the ligated lesion was lifted carefully. Next, a 5-mm hole was made in the center of an absorptive sheet measuring $0.15 \mathrm{~mm}$ in thickness and containing polyglycolic acid (PGA) (Neoveil; Gunze Ltd., Kyoto, Japan). The ligated bullous lesion was then pulled through the cut hole, and the lesion was resected by endostapling across the PGA sheet. After firing the endostapler, we performed a sealing test by inflating the lung under $15 \mathrm{~cm}$ of water pressure to detect persistent air leaks, and $3 \mathrm{~mL}$ of autologous blood was placed by drops onto the sheet as an autologous blood patch. An $8-\mathrm{Fr}$ drainage tube was inserted through the same puncture hole 
Table 1 Cases operated on using the jellyfish method for spontaneous pneumothorax

\begin{tabular}{|c|c|c|c|c|c|c|c|}
\hline Case & Age (years) & Gender & Side & $\begin{array}{l}\text { Location of } \\
\text { bullous lesion }\end{array}$ & $\begin{array}{l}\text { Operation } \\
\text { time (min) }\end{array}$ & $\begin{array}{l}\text { Duration of postoperative } \\
\text { drainage (days) }\end{array}$ & $\begin{array}{c}\text { Postoperative } \\
\text { hospital stay (days) }\end{array}$ \\
\hline 1 & 16 & Male & Left & $\mathrm{S} 1$ & 92 & 1 & 3 \\
\hline 2 & 21 & Female & Right & S1, S6, S8 & 113 & 3 & 4 \\
\hline 3 & 17 & Male & Left & $\mathrm{S} 1$ & 80 & 1 & 2 \\
\hline 5 & 19 & Male & Right & S1 & 75 & 1 & 3 \\
\hline 6 & 19 & Male & Right & S1 & 75 & 1 & 3 \\
\hline
\end{tabular}

under thoracoscopic guidance.

We performed the jellyfish method electively in 6 patients [ 4 men and 2 women; mean age: 18 years (range: 16-21 years)] from April to December 2016 (Table 1). All underwent the procedure successfully without conversion to open thoracotomy or any mortalities. Except for Case No. 2, the emphysematous bullous lesion was localized in the apex of the affected lung, and the lesions were resected in all within two firings of the endostapler. The mean duration of operation with the jellyfish method was $92 \mathrm{~min}$ (range, 75-113 $\mathrm{min}$ ). Except for Case No. 2, the duration of postoperative drainage was within $24 \mathrm{~h}$. None of the patients had a postoperative ipsilateral recurrence of pneumothorax or empyema during the postoperative follow-up period (median: 14 months, range: 11-16 months).

\section{Comments}

Spontaneous pneumothorax is now commonly treated with conventional three-port VATS, which is associated with less pain and a shorter hospital stay and improved cosmetic results and overall patient satisfaction compared with thoracotomy (8). However, pain or paraesthesia due to intercostal nerve compression during conventional three-port VATS is often seen (9). Technical advances in thoracoscopic instruments have allowed for the modification of conventional VATS to reduced-port thoracoscopic surgery (1-3). In reduced-port VATS, endoscopic instruments such as graspers, dissectors, staplers and the camera are inserted into the thoracic cavity through a single port. Reduced-port VATS involves only one intercostal space instead of three, thereby reducing the surgical trauma and postoperative pain and speeding recovery and return to work. However, the postoperative recurrence rates and ways of preventing recurrence have not been well-discussed for reduced-port thoracoscopic bullectomy.

In conventional three-port VATS, postoperative recurrence has been reported in approximately $10 \%$ of patients (4). Many surgeons have suggested that an additional coverage procedure on the staple-line might be useful for preventing postoperative recurrence after thoracoscopic bullectomy (5). Saito et al. reported a covering technique involving resection by endostapling across the covering PGA sheet (6). The main advantage of this technique is that the covering sheet was able to be securely fixed around the staple line, thereby avoiding the use of bioglue. We modified this method in our reducedport VATS strategy (jellyfish method). The thin puncture device (Endo Close) was very useful for covering the staple-line while performing reduced-port thoracoscopic bullectomy. The device has always been used in laparoscopic surgery to close the abdominal wound. The stylet retracts as the needle is pushed through the chest wall, and it has a notched end that is used to capture and hold the suture. Therefore, the present technique can be performed quickly with good results in reduced-port VATS (shown as Table 1) given the ease of capturing the endoloop and introducing the covering sheet though the bullous lesion (shown as Figures 1 and 2).

Another important factor for promising outcomes is preventing prolonged postoperative air leak. Usually, bioglue is used in VATS to prevent postoperative air leak and dislocation of covering materials (9). However, bioglue carries a risk of transmission of infectious pathogens. Autologous blood patch pleurodesis has been reported to be effective for plugging persistent air leaks (10). We performed this patching by placing autologous blood drops onto the sheet after endostapling. In our series, 5 of the 6 patients were able to have their drainage tube removed 
within $24 \mathrm{~h}$ after surgery. An autologous blood patch after endostapling may also be effective for preventing prolonged postoperative air leak in reduced-port thoracoscopic bullectomy.

The reduced economic cost associated with reducedport thoracoscopic surgery is another promising benefit. Recently, technological advances in VATS have resulted in the development of several new instruments (11). However, the instruments required for reduced-port VATS are expensive, making our technique somewhat costly. The absorptive sheet and devices cost 8,400 yen (\$93 US) and 9,800 yen ( $\$ 100 \mathrm{US}$ ), respectively. However, this cost might be reasonable, given that our technique requires less bioglue and other expensive thin instruments and results in less pain than conventional three-port VATS.

There are several limitations to our new technique. First, our approach may only be useful for localized bullous lesions (within two firings of endostapling). One reason for this is the difficulty of complete resection for extensive bullous lesion when the lesion is covered with PGA sheet. In addition, the fixed covering sheet has limited mobility. Adequate covering of extensive bullous lesions may be difficult using our method. Second, all cases had a relatively short postoperative follow-up period (within 14 months). The long-term efficacy of our technique in reducing the postoperative recurrence rates therefore remains unclear. Third, there were too few cases for analyses, so our data should be interpreted carefully. The further accumulation of clinical experience is needed.

In conclusion, we found the jellyfish method to be a simple and easy technique for the wide reinforcement of the staple line in reduced-port VATS for spontaneous pneumothorax. Our new technique may be effective for reducing the postoperative recurrence rates in reduced-port thoracoscopic bullectomy.

\section{Acknowledgements}

None.

\section{Footnote}

Conflicts of Interest: The authors have no conflicts of interest to declare.

Informed Consent: Written informed consent was obtained from all patients.

\section{References}

1. Ng CS, Rocco G, Wong RH, et al. Uniportal and single-incision video-assisted thoracic surgery: the state of the art. Interact Cardiovasc Thorac Surg 2014;19:661-6.

2. Yang Y, Dong J, Huang Y. Single-incision versus conventional three-port video-assisted surgery in the treatment of pneumothorax: a systematic review and meta-analysis. Interact Cardiovasc Thorac Surg 2016;23:722-8.

3. Son BS, Kim DH, Lee SK, et al. Small Single-Incision Thoracoscopic Surgery Using an Anchoring Suture in Patients With Primary Spontaneous Pneumothorax: A Safe and Feasible Procedure. Ann Thorac Surg 2015;100:1224-9.

4. Sawada S, Watanabe Y, Moriyama S. Video-assisted thoracoscopic surgery for primary spontaneous pneumothorax: evaluation of indications and long-term outcome compared with conservative treatment and open thoracotomy. Chest 2005;127:2226-30.

5. Lee S, Kim HR, Cho S, et al. Staple line coverage after bullectomy for primary spontaneous pneumothorax: a randomized trial. Ann Thorac Surg 2014;98:2005-11.

6. Saito T, Kaneda H, Konobu T, et al. The covering with forceps-assisted polymeric biodegradable sheet and endostapling method: a simplified technique for wide coverage and reinforcement of staple-line in videoassisted thoracoscopic bullectomy for spontaneous pneumothorax. Interact Cardiovasc Thorac Surg 2011;12:103-5.

7. Yasuda M, Nakanishi R, Mori M, et al. The jellyfish method. Performing thoracoscopic bullectomy using the jellyfish method for Case No. 1. Asvide 2017;4:517. Available online: http://www.asvide.com/ articles $/ 1836$

8. Goto T, Kadota Y, Mori T, et al. Video-assisted thoracic surgery for pneumothorax: republication of a systematic review and a proposal by the guideline committee of the Japanese association for chest surgery 2014. Gen Thorac Cardiovasc Surg 2015;63:8-13.

9. Yasuda M, Nagashima A, Haro A, et al. How should synchronous multiple primary adenocarcinomas of the lung be resected? Ann Thorac Surg 2014;97:e151-3.

10. Cao Gq, Kang J, Wang F, et al. Intrapleural instillation of autologous blood for persistent air leak in spontaneous pneumothorax in patients with advanced chronic 
obstructive pulmonary disease. Ann Thorac Surg 2012;93:1652-7.

11. Chen JS, Hsu HH, Kuo SW, et al. Needlescopic versus

Cite this article as: Yasuda $M$, Nakanishi R, Mori M, Ashikari S, Oyama T, Hanagiri T. Simple covering technique in thoracoscopic bullectomy for spontaneous pneumothorax. J Thorac Dis 2017;9(11):4584-4588. doi: 10.21037/ jtd.2017.10.95 conventional video-assisted thoracic surgery for primary spontaneous pneumothorax: a comparative study. Ann Thorac Surg 2003;75:1080-5. 